The data in Fig. 1 are based mostly on Venus signals of the atmospherics-like variety (class I), since the class II signals are not observed so frequently. With reference to the latter signals, a recent additional observation is that the pulse-like class II signals occur over a frequency-band of at least 2-4 megacycles per sec. with a given pulse being received earlier at higher frequencies, the dispersion in time being about 2 sec. per megacycle per sec. of frequency difference.

Radio Observatory,

JoHN D. KRAUS

Department of Electrical Engineering, Ohio State University,

Columbus 10, Ohio. July 23.

1 Kraus, J. D., Nature, 178, 33, 103, 159 (1956).

${ }^{2}$ Ross, F. E., Astrophys. J., 68, 57 (1928).

${ }^{3}$ Kraus, J. D., Sky and Telescope, 15, 358 (1956).

\section{The Second Purple Light}

BY photoelectric observation of the twilight sky, Bigg' has found evidence of scattering of the Sun's rays from an elevated atmospheric layer. Assuming primary scattering, he calculates its height to average some $81 \mathrm{~km}$. and finds an association between the intensity of the effect and the incidence of meteor showers. He also observed scattering by a lower dust layer (10-25 km.), which is the cause of the purple light frequently observed above the western horizon after sunset and before sunrise. But after some great volcanic eruptions a striking feature of the brilliant sunsets has been the long-continued after-glows, with a second purple light appearing some time after the first had set and only fading away some $1 \frac{1}{2}-2 \mathrm{hr}$. after sunset.

It is interesting to recall, in the light of Bigg's work, the discussion which took place in Nature after the great Krakatoa eruption in August 1883 as to the origin of this second purple light-a discussion continued and amplified in the Report of the Krakatoa Committee of the Royal Society published in 1888. In the first place, $R$. von Helmholtz ${ }^{2}$ suggested that primary scattering of light from dust at heights around $70 \mathrm{~km}$. was the cause of the second purple light; but this view did not gain general acceptance and it was instead held that both purple lights were caused by the lower dust layer. This explanation is still current-Neuberger", for example, says "the purple light is sometimes sufficiently intense to cause a secondary purple light for an observer situated farther on the night side of the earth". The primary scattering hypothesis was rejected mainly owing to the " $a$ priori improbability that the reflecting stratum, whether derived from cosmical or from terrestrial sources, should occur in two definite layers" (Archibald $\left.{ }^{4}\right)$. Now that we know more of the complexities of the atmosphere and, in particular, of the strong temperature inversion with its base at about $80 \mathrm{~km}$., this argument fails, and Helmholtz's explanation seems much the more probable. The relative intensity of the purple lights seems more in keeping with a two-layer theory; a secondary effect of the first purple light would not be expected to have as great intensity as reported in 1883 and 1884 .

If we assume primary scattering from a high-level dust layer, then six observations of the second purple light by Riccò ${ }^{4}$ at Palermo give a mean height of $85 \mathrm{~km}$. and others by Brauner ${ }^{5}$ at Prague about
$80 \mathrm{~km}$. These values compare well with Bigg's mean of $81 \mathrm{~km}$.; so it seems very probable that Krakatoa dust was held in suspension at these heights over a period of some months.

Division of Meteorological Physics.

E. L. DEACON

Commonwealth Scientific and

Industrial Research Organization, Melbourne.

May 9.

${ }^{1}$ Bigg, E. K., Nature, 177, 77 (1956).

2 Helmholtz, R, von, Nature, 29, 130 (1883).

3 Neuberger, H., "Compendium of Meteorology", 61 (Amer. Met. Soc. Boston, Mass., 1951).

"Archibald, E. D., Part 4, Sect. 4, "The Eruption of Krakatoa", Rep. Krakatoa Cmttee. of Roy. Soc. (Trübner, London, 1888).

5 Brauner, B., Nature, 29, 223 (1884).

\section{Physical Constants in Extra-Galactic Nebulæ}

RECENT wave-length measurements by $R$. Minkowski and O. C. Wilson ${ }^{1}$ of spectral lines of $\mathrm{H}, \mathrm{N}$ II, O I, O II, Ne III and $\mathrm{Ne} \mathrm{V}$ for the colliding galaxies identified with the radio source Cygnus $A$ permit comparison of galaxy values of physical constants with local values. In particular, radio measures by A. E. Lilley and E. F. McClain ${ }^{2}$, in combination with the optical measures, permit estimates on the ratio of galactic to local values of the fine structure constant, $\alpha$, and the proton gyromagnetic ratio, $g$. The observed system is estimated to be $3 \times 10^{8}$ light years from us on the Humason, Mayall, Sandage scale $^{3}$.

In the discussion of these measures, we shall be concerned with a gross red shift, assumed of the form $\Delta \lambda / \lambda=$ constant, upon which may be superimposed changes in fine and hyperfine structure. It is not necessary to decide whether the gross red shift arises from a change of constants or from a velocity shift. It is further assumed that the interaction Hamiltonian for atomic spectra is of the form:

$$
H=H_{0}+\alpha^{2} H_{f}+g \frac{m}{M} \alpha^{2} H_{h f}
$$

Among these terms, $H_{0}$ arises from electrostatic interactions, $H_{f}$ from relativistic effects usually represented by spin-orbit interactions, and $H_{h f}$ in this specific case is the spin-spin interaction as in the hydrogen hyperfine structure. If we use the ionization energy of hydrogen as the energy unit, we obtain from equation (1) the quantity $h c R=\frac{1}{2} \alpha^{2} m c^{2}$.

In writing equation. (1) it is assumed that both fine structure and hyperfine structure are small perturbations, and especially for the former that Russell-Saunders coupling predominates (see Condon and Shortley ${ }^{4}$ and Bethe ${ }^{5}$ ). Note particularly that the ratio of the fine and hyperfine interaction energies to the electrostatic interaction energies depends upon no other physical constants except those indicated in equation (1).

From measures of all available lines, Minkowski and Wilson conclude that for the optical Cygnus $A$ the quantity $c \Delta \lambda / \lambda=16,805 \pm 6 \mathrm{~km}$. $/ \mathrm{sec}$. The fine structure produces the doublet splitting of the terms ${ }^{3} P_{n}-{ }^{1} D$. Of the three doublets measured, the measures of OI appear to suffer from blends and only those of N II and Ne III will be discussed. The doublet separations are taken proportional to $(1+\Delta \lambda / \lambda) \alpha^{2}$. With $\Delta \lambda / \lambda$ as above, we obtain from the data: 